(2) Open Access Full Text Article

ORIGINAL RESEARCH

\title{
Association between angiogenesis and cytotoxic signatures in the tumor microenvironment of gastric cancer
}

This article was published in the following Dove Press journal:

OncoTargets and Therapy

\author{
Yi Feng ${ }^{1,2}$ \\ Ying Dai ${ }^{1,2}$ \\ Zhihua Gong ${ }^{1,2}$ \\ Jia-Nan Cheng ${ }^{1,2}$ \\ Longhui Zhang ${ }^{1,2}$ \\ Chengdu Sun ${ }^{1,2}$ \\ Xianghua Zeng ${ }^{1,2}$ \\ Qingzhu Jia ${ }^{1,2}$ \\ Bo Zhu ${ }^{1,2}$
}

'Institute of Cancer, Xinqiao Hospital, Third Military Medical University, Chongqing, People's Republic of China; ${ }^{2}$ Chongqing Key Laboratory of Tumor Immunotherapy, Chongqing, People's Republic of China
Correspondence: Bo Zhu Institute of Cancer, Xinqiao Hospital, I 84 Xinqiao Street, Shapingba District, Chongqing, People's Republic of China Tel +86 I35 946I I534 Email bo.zhu@tmmu.edu.cn
Background: A suppressive immune microenvironment and pathological angiogenesis are hallmarks of gastric cancer. Theoretically, immune checkpoint inhibitors (ICIs) stimulate preprimed neoantigen-specific $\mathrm{T}$ cells, and antiangiogenic agents then facilitate their infiltration into the tumor niche by promoting vascular normalization. Currently, the interconnections of these two phenotypes and their relevance to the tumor microenvironment (TME) have not been fully characterized in gastric cancer.

Materials and methods: Transcriptome profiling data retrieved from The Cancer Genome Atlas (TCGA) database were used to deconvolute the feature of TME for gastric cancer $(\mathrm{N}=375)$. Machine learning, correlation, and prognosis analysis were applied to elucidate the correlations between angiogenesis, cytotoxic T lymphocyte infiltration, and patient survival.

Results: Substantial heterogeneous infiltration of immune cell populations among cases was observed. Furthermore, among targetable pathways, angiogenesis was identified as the dominant factor in discriminating different infiltration statuses. Most importantly, the angiogenesis pathway was negatively correlated with the amount of activated $\mathrm{CD} 8^{+} \mathrm{T}$ cells only for patients with a higher infiltration, and the concomitance of low angiogenesis signaling and highly activated CD8 ${ }^{+} \mathrm{T}$-cell infiltration was associated with a significant survival benefit.

Conclusion: Our findings demonstrated a negative correlation between angiogenesis signaling and cytotoxic function in gastric cancer patients with a highly infiltrated immune niche. These data provided a rationale for potential combination strategy and further clinical investigations of ICIs plus antiangiogenesis agents for patients with gastric cancer with an inflamed TME.

Keywords: gastric cancer, immune microenvironment, TCGA, angiogenesis, therapeutic implications

\section{Introduction}

Gastric cancer is the fifth most common malignant disease and the third leading cause of cancer-related mortality worldwide. ${ }^{1}$ However, the clinical benefit of available treatment strategies is limited, emphasizing the need to develop novel strategies to improve disease outcomes. Cancer immunotherapy has made encouraging breakthroughs in a variety of other solid tumors, ${ }^{2}$ which has also been evaluated for use as a treatment for gastric cancer recently. In Phase Ib KEYNOTE-012 trial, researchers found prolonged survival in advanced gastric cancer patients treated with pembrolizumab, an anti-programmed cell death-1 (PD-1) antibody, ${ }^{3}$ and further identified upregulation of interferon- $\gamma$-related genes, including STAT1, HLA-DRA, IFNG, IDO1, and CXCL9/10, in the tumor microenvironment (TME) of responders. ${ }^{3}$ This phenomenon suggests that successful stimulation of the immunological suppressive TME requires 
pre-primed and infiltrated neoantigen-specific T cells in the tumor niche to eliminate the mutated cells. Therefore, the prognostic value of these immune signatures provides a convincing rationale for exploring combination therapies to improve the objective response rate (ORR) by promoting immune cell infiltration.

Emerging evidence suggests that impaired infiltration of cytotoxic T cells (CTLs) in the TME may be related to the increased level of vascular endothelial growth factor A (VEGFA), ${ }^{4}$ which facilitates vascularization without wellregulated expression of adhesion molecules, such as intercellular adhesion molecule-1 (ICAM-1) and vascular cell adhesion-1 (VCAM-1). The AVAGAST trial demonstrated that the addition of bevacizumab, a VEGFA-directed monoclonal antibody, to the standard chemotherapy increased the percentage of patients achieving an ORR and slightly prolonged progression-free survival (PFS) and overall survival (OS). ${ }^{5}$ Another antagonist targeting VEGFR-2, ramucirumab, was also shown to provide some clinical benefits as monotherapy or in combination with paclitaxel as a second-line regimen in REGARD $^{6}$ and RAINBOW ${ }^{7}$ trials. Moreover, administration of apatinib, a VEGFR tyrosine kinase inhibitor, was associated with improved PFS and OS as third-line or later treatment. ${ }^{8}$ The favorable effects of antiangiogenic agents in patients with metastatic gastric cancer are thought to be related to reduce blood supply to tumor cells rather than immune modulation. Elucidating the relationships between angiogenesis and cytotoxic functions would expand our understanding of the immune microenvironment of gastric cancer and provide a basis for further investigations of combination therapies.

In this study, we aimed to elucidate the immune landscape of gastric cancers by analyzing previous clinical cases reported in The Cancer Genome Atlas (TCGA) project. We identified several dominant immunological features involved in determining local immune cell infiltration status. Among these features, angiogenesis, the most important drugtargetable signaling pathway, was significantly correlated with the magnitude of the signature of cytotoxic function, particularly in patients with highly infiltrated TME. Therefore, our study provided a rationale for potential combination therapeutic strategies and further clinical investigations of combination therapies.

\section{Materials and methods}

\section{Data sources}

The RSEM-normalized RNA sequencing data for the stomach adenocarcinoma (STAD) cohorts were downloaded from TCGA Data Portal (https://gdc-portal.nci.nih.gov/). All gastric cancers analyzed in this study were untreated primary lesions $(\mathrm{N}=375)$. Additional data sets were also downloaded from TCGA in May 2017. The Hallmark gene sets were downloaded from GSEA Molecular Signatures Database v6.0 (http://software.broadinstitute.org/gsea/ msigdb/index.jsp).

\section{Gene signatures and single-sample gene set enrichment analysis (ssGSEA) scores}

The levels of immune infiltration were quantified using a previously validated computational method based on expression profiles of immunomodulatory factors from 28 immune cell types, comprising 782 genes in total. ${ }^{9}$ The degree of immune infiltration was determined by the ssGSEA, ${ }^{10}$ computed using R-package Gene Set Variation Analysis (GSVA). Cytotoxic (CYT) activity was computed as the geometric mean of expression levels of PRF1 and GZMA, as previously reported. ${ }^{11}$ For antigen-presenting machinery (APM), we used a seven-gene signature composed of MHC class I genes and antigen-processing genes. ${ }^{12}$ The IFN- $\gamma$ signature was calculated as the mean of a series of gene profiles as described in KEYNOTE-059, ${ }^{13}$ CheckMate-275, ${ }^{14}$ KEYNOTE-012, ${ }^{3}$ and POPLAR ${ }^{15}$ clinical trials. The angiogenesis and $\mathrm{Wnt} / \beta$-catenin pathway were calculated using ssGSEA scores from HALLMARK_ANGIOGENESIS and HALLMARK_WNT_BETA_CATENIN_SIGNALING.

\section{Identification of determinants of the tumor immune microenvironment}

Cases of high infiltration and low infiltration groups were selected for examination. A random forest classifier, including 42 parameters from the Hallmark gene set, was trained to separate the group of cases with high infiltration levels from cases with low infiltration levels using the R package randomForest with 10,000 trees. Eight signaling pathways of Hallmark that directly characterized the inflammation were excluded (TNFA_SIGNALING_VIA_NFKB, IL6_JAK_STAT3_SIGNALING, INTERFERON_ALPHA_RESPONSE, INTERFERON_ GAMMA_RESPONSE, HALLMARK_COMPLEMENT, INFLAMMATORY_RESPONSE, IL2_STAT5_SIGNALING, and ALLOGRAFT_REJECTION). The mean decrease in Gini over all out-of-bag cross-validated predictions was applied to rank the predictors. The immunophenogram was visualized as previously described. ${ }^{16}$ The aforementioned infiltration signatures of 28 immune cell gene profiles were selected for multidimensional scaling (MDS) analysis. Sample-wise Z-scores from gene expression data were calculated for cell types, and an average $Z$-score from the corresponding gene sets was calculated. 


\section{Statistical analysis}

Sample sizes from available TCGA data were regarded as adequate according to power estimates performed previously using equivalent tests. ${ }^{17}$ Two-sided Student's $t$-test and one-way analysis of variance (ANOVA) were used for comparisons using the $\mathrm{R}$ package. Unsupervised clustering for tumor cases, immune cell types, and gene sets was performed with hierarchical clustering using Pearson's correlation. Furthermore, Pearson's correlation coefficients were also reported for ssGSEA scores in the angiogenesis pathway, Wnt/ $\beta$-catenin signaling pathway, and immune signatures. For survival analysis, $p$-values in Figure 4 were obtained from Cox proportional-hazards regression models using the R package survival. Kaplan-Meier curves were generated using the survfit function from the survival package.

\section{Ethical approval and informed consent}

This article does not contain any studies with human participants or animals performed by any of the authors.

\section{Results \\ Gastric cancers exhibited heterogeneous immune cell infiltration}

To evaluate immune cell infiltration in the TME of gastric cancer, we analyzed the RNA-sequencing profiles retrieved from TCGA. ssGSEA was introduced to deconvolute the relative infiltration level into a normalized ssGSEA score. ${ }^{10}$ Significant heterogeneous infiltration of diverse immune cell populations was identified by visualizing the calculated scores (Figure 1A and Table S1). Based on their infiltration spectrum, all cases were classified into high, median, or low immune infiltration groups by an unsupervised classification method. The high infiltration group seemed to have more immune-active cells, containing activated $\mathrm{T}$ cells $\left(\mathrm{T}_{\mathrm{ACT}} \mathrm{s}\right)$, central memory $\mathrm{T}$ cells, effector memory $\mathrm{T}$ cells, natural killer $\mathrm{T}$ cells, type $1 \mathrm{~T}$ helper cells (Th1), T follicular helper cells, $\gamma \delta \mathrm{T}$ cells, activated $\mathrm{B}$ cells, and activated dendritic cells (Figure S1A). With the higher levels of activated subtypes, immune-suppressive subtypes, such as myeloid-derived suppressor cells (MDSCs)

A
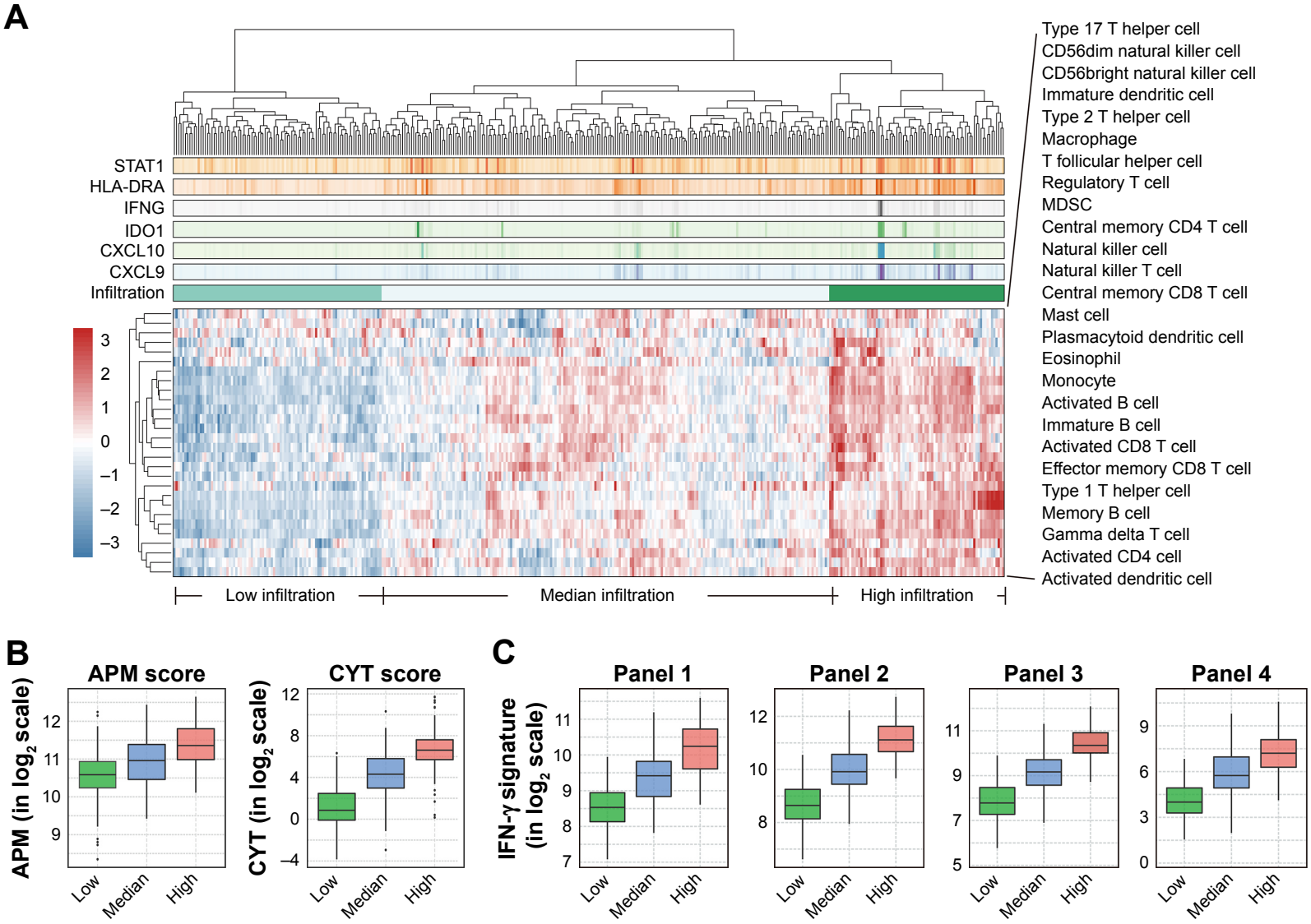

Figure I Heterogeneous infiltration landscape of gastric cancers.

Notes: (A) ssGSEA analysis of 375 gastric cancer cases identifying relative scores of infiltrated immune cell subtypes clustered into high, median, and low infiltration phenotypes. (B) Relative APM and CYT scores among different groups. (C) IFN- $\gamma$ signatures based on gene panels described in KEYNOTE-059 (panel I), CheckMate-275 (panel 2), KEYNOTE-0I2 (panel 3), and POPLAR (panel 4) clinical trials among different groups. All box plots represent values within the IQR. Outliers are plotted as values $>I .5 \times$ IQR (circles). All $p$-values for significance $(<0.05)$ were obtained from two-tailed Student's $t$-test and one-way ANOVA for continuous comparisons $(\mathrm{N}=375)$. Abbreviations: ssGSEA, single-sample gene set enrichment analysis; APM, antigen-presenting machinery; CYT, cytotoxic; IQR, interquartile range; ANOVA, analysis of variance. 
and regulatory $\mathrm{T}$ cells (Tregs), were also enriched in the high infiltration group (Figure S1A), implying a negative feedback in modulating the cytotoxic response.

To thoroughly characterize the immunophenotypes of distinct groups, with a particular focus on cytotoxic function, we compared levels of several representative immunomodulatory and effector molecules, including APM, CYT scores, ${ }^{11}$ and gene panels of IFN- $\gamma$ signatures, including panels applied in KEYNOTE-059 $9^{13}$ (panel 1), CheckMate-275 ${ }^{14}$ (panel 2), KEYNOTE-0123 (panel 3), and POPLAR ${ }^{15}$ (panel 4). We observed enhanced cytotoxic function in the high infiltration group, characterized by elevated APM, CYT score, and the IFN- $\gamma$ signature of different standards (Figure 1B and $\mathrm{C}$ and Table S2), strongly suggesting high cytotoxic potential in patients with gastric cancer exhibiting higher immune cell infiltration.

Additionally, we compared the aforementioned signatures among different pathologic subtypes and found no significant difference between any two distinct types (Figure S1B). This suggested that infiltration levels were not subject to pathologic type.

\section{Machine learning identified angiogenesis as a predominant determinant of immune cell infiltration}

To identify the most dominant determinants of immune cell infiltration in gastric cancer, we introduced a machine learning approach (random forest), based on a multitude of decision trees. ${ }^{18}$ A total of 42 different input variables from the Hallmark database were included to build the decision trees for discriminating the high infiltration versus low infiltration status. Eight signaling pathways that directly characterized the inflammation were excluded because they were usually indicators but not the original reason for immune signatures of TME. By MDS visualization, the similarities in immunophenotypes of every two cases were indicated as their spatial distance (Figure 2A). The most dominant variables were identified by sorting the importance of each pathway (Figure 2B). There was a huge gap between the first two elements (KRAS and apoptosis) and the remaing factors. As for KRAS, it has been reported that expression of a mutant KRAS oncogene could induce strong responses by activating cytokines and $\mathrm{T}$ cells and thereby potently influence the immune milieu. ${ }^{19,20}$ As for apoptosis, its positive correlations with host immunity have also been confirmed by several studies. ${ }^{21,22}$ Interestingly, angiogenesis pathway was identified as the most important variable among the drug-targetable signaling pathway (Figure 2B). However, the Wnt/ $\beta$-catenin pathway, which is thought to play a critical role in hindering T-cell infiltration in patients with melanoma, ${ }^{23}$ was not ranked high in the TME of gastric cancer. These findings suggested tumor-type-specific regulation of immune cell infiltration and highlighted the role of angiogenesis in forming the immune microenvironment in gastric cancer.
A

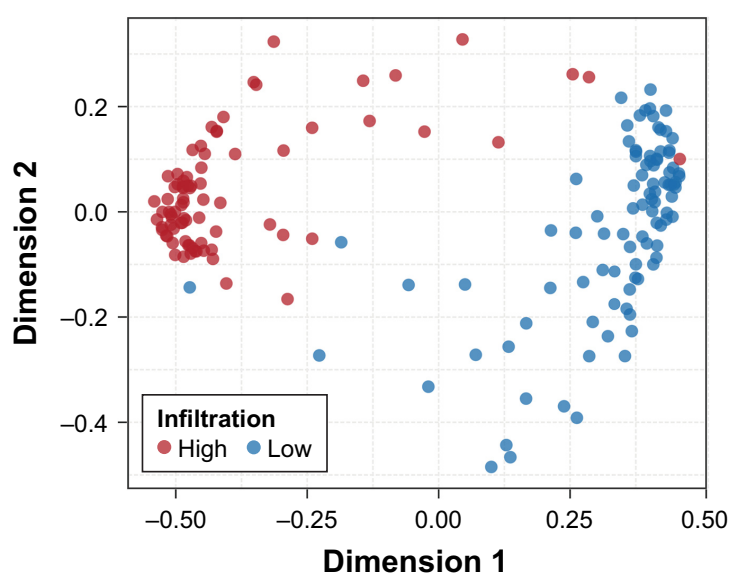

B

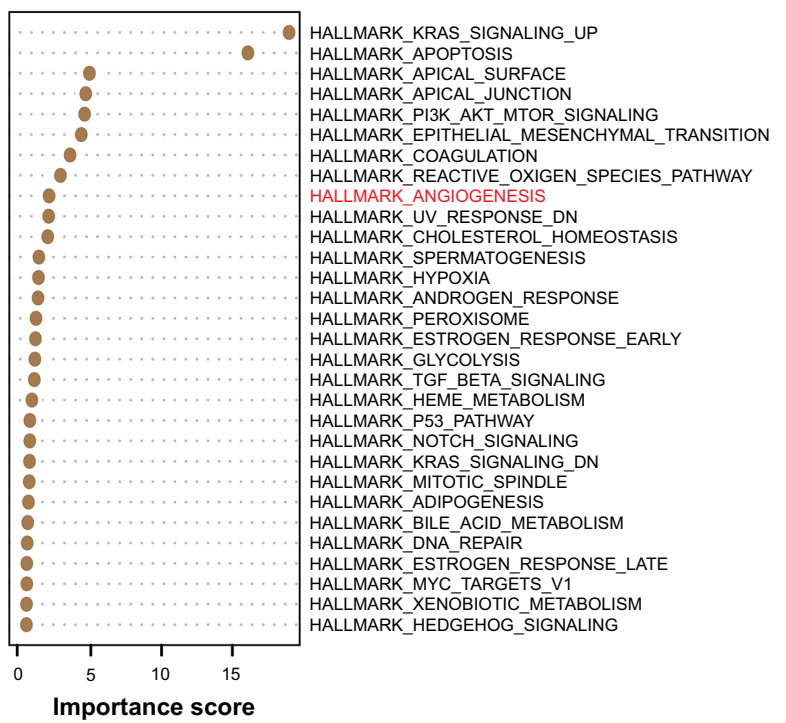

Figure 2 Determinants of immune characterization in gastric cancers.

Notes: (A) Visualization based on two-dimensional coordinates from MDS of expression profiles from the gene sets used to categorize immune infiltration in gastric cancers. (B) The first 30 determinants of immune signatures identified by the machine learning approach. The importance of each parameter was sorted according to the mean decrease in Gini.

Abbreviation: MDS, multidimensional scaling. 


\section{Angiogenesis, but not the $\mathrm{Wnt} / \beta$-catenin signaling pathway, was associated with cytotoxic function in TMEs exhibiting high infiltration}

Previous studies have found that VEGF/VEGFR inhibitors might repolarize immune cells to the Th1 phenotype, resulting in enhanced antitumor effects. ${ }^{24}$ Here, we analyzed the association between angiogenesis and cytotoxic function in the TME of a large cohort of gastric cancers. CYT scores and the infiltration of $\mathrm{CD}^{+} \mathrm{T}_{\mathrm{ACT}} \mathrm{S}$ were examined as indicators of cytotoxic function due to their ultimate effective roles in tumor immunity. When the correlations were tested for the entire cohort, we found an almost irrelevant correlation between the angiogenesis pathway and cytotoxic function, which was not represented by the CYT score or the infiltration of $\mathrm{CD}^{+} \mathrm{T}_{\mathrm{ACT}} \mathrm{S}$ (Figure $3 \mathrm{~A}$ and Table $\mathrm{S} 3$ ). However, when comparisons for each infiltration status were applied separately, prominent negative associations between angiogenesis and CYT scores were found, particularly for highly infiltrated TME (Figure 3A). A similar tendency was also observed between angiogenesis and $\mathrm{CD}^{+} \mathrm{T}_{\mathrm{ACT}} \mathrm{S}$ (Figure $3 \mathrm{~A}$ and Table S3). Immune-inhibitory cell types, such as MDSCs and Tregs, showed positive correlations (Figure 3A and Table S3), which is consistent with previous reports. ${ }^{25,26}$

As mentioned earlier, the $\mathrm{Wnt} / \beta$-catenin signaling pathway has been validated to regulate the development of CTLs, the activation of Tregs, and further influence antitumor immunity in malignant melanoma. ${ }^{23,27}$ However, we observed no significant correlations between $\mathrm{Wnt} / \beta$-catenin signaling and infiltration characteristics in gastric cancer (Figure S2A and $\underline{B}$ and Table S4 ), which is consistent with the findings in Figure 2B and implied a tumor-type-dependent mechanism of tuning immune cell infiltration.

Based on these findings, we then analyzed the relationships between angiogenesis and IFN- $\gamma$ signature in detail using several clinically tested gene panels. Our exploration revealed interesting results (Figure 3B and Table S5): 1) there were almost no correlations between angiogenesis and IFN- $\gamma$ signature when judged at the integral level; 2 ) in the low infiltration group, angiogenesis was irrelevant to IFN- $\gamma$ signature; and 3 ) inverse correlations with angiogenesis were only observed in the high and medium infiltration groups. Considering the valuable significance of the IFN- $\gamma$ signature in response prediction of immune checkpoint inhibitors (ICIs), these data indicated that the angiogenesis pathway, but not the $\mathrm{Wnt} / \beta$-catenin signaling pathway, was inversely related to the responsiveness to immunotherapy, particularly with inflamed immune niche, supporting further clinical investigations of targeting angiogenesis to improve clinical outcomes of immunotherapy by enhancing immune cell infiltration and ameliorating immune suppression.

\section{Favorable survival predicted by angiogenesis and infiltration of activated $C D 8^{+} \mathrm{T}$ cells}

Higher infiltration of immune-activating cells, especially $\mathrm{CD}^{+} \mathrm{T}_{\mathrm{ACT}} \mathrm{s}$, is usually associated with improved responsiveness to immunotherapy and always results in better prognosis. ${ }^{28}$ Therefore, we compared the clinical outcomes between two cohorts grouped by the infiltration of $\mathrm{CD}^{+} \mathrm{T}_{\mathrm{ACT}} \mathrm{S}$ $\left(A c t C D 8^{\mathrm{Hi}}\right.$ and $\mathrm{ActCD} 8^{\mathrm{Lo}}$ ). Unexpectedly, the ActCD ${ }^{\mathrm{Hi}}$ group did not show significantly prolonged survival compared with the ActCD8 ${ }^{\text {Lo }}$ group (Figure 4A and Table S6), indicating that $\mathrm{CD}^{+} \mathrm{T}_{\mathrm{ACT}} \mathrm{s}$ infiltration might not be an effective predictor of prognosis in gastric cancer. We then checked the correlation between angiogenesis and prognosis. Interestingly, better prognoses in the $\mathrm{ANGIO}^{\mathrm{Lo}}$ group were observed in both high and median infiltration subpopulations, but not in the low infiltration subpopulation (Figure 4B and Table S6). These data suggested that angiogenesis participated as a negative predictor of prognoses in patients with gastric cancer, particularly in those with inflamed immune characterization.

From findings, we hypothesized that lower angiogenesis and higher infiltration of $\mathrm{CD}^{+} \mathrm{T}_{\mathrm{ACT}} \mathrm{s}$ cells may be favorable for prolonging survival. To test this, cases were categorized into four groups (ActCD $8{ }^{\mathrm{Hi}} \mathrm{Angio}^{\mathrm{Hi}}$, ActCD $8^{\mathrm{Hi}} \mathrm{Angio}^{\mathrm{Lo}}$, ActCD $8^{\mathrm{Lo}}$ Angio ${ }^{\mathrm{Hi}}$, and $\operatorname{ActCD} 8^{\mathrm{Lo}}$ Angio ${ }^{\mathrm{Lo}}$ ) based on the ssGSEA scores of $\mathrm{CD}^{+} \mathrm{T}_{\mathrm{ACT}} \mathrm{s}$ and angiogenesis. As for the high and median infiltration groups, a comparison of the prognoses of the four groups showed that ActCD $8{ }^{\mathrm{Hi}} \mathrm{Angio}^{\mathrm{Lo}}$ patients exhibited better prognoses than patients in other groups (Figure 4C and Table S6). On the contrary, in the low infiltration group, ActCD $8^{\mathrm{Hi}} \mathrm{Angio}^{\mathrm{Lo}}$ was not inclined to exhibit prolonged survival compared to other subpopulations and even showed inverse trends (Figure 4C and Table S6). These data provided insights into further testing of immunotherapy/antiangiogenic combination therapy in the clinical setting.

\section{Discussion}

Major breakthroughs in immunotherapy have recently been made in various solid tumors, while several hurdles have been met in gastric cancer. Early clinical trials on tremelimumab only observed disappointing results for metastatic gastric adenocarcinomas. ${ }^{29}$ Soon after, compelling data 
A

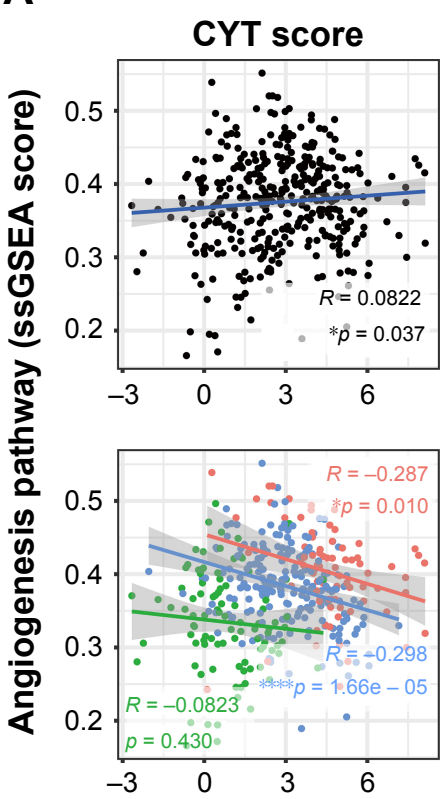

Activated CD8 T cells (ssGSEA score)
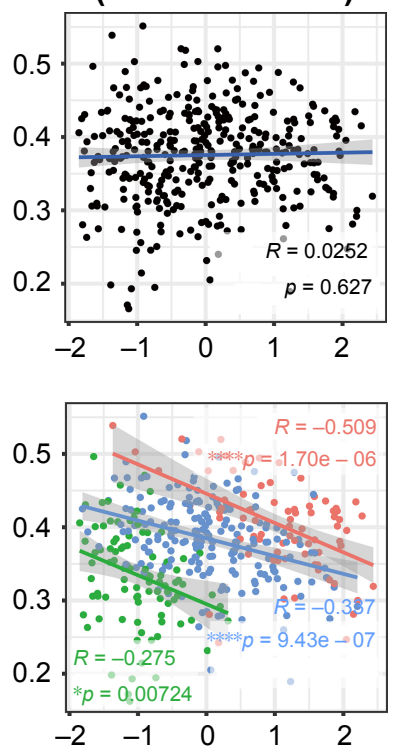

MDSC (ssGSEA score)
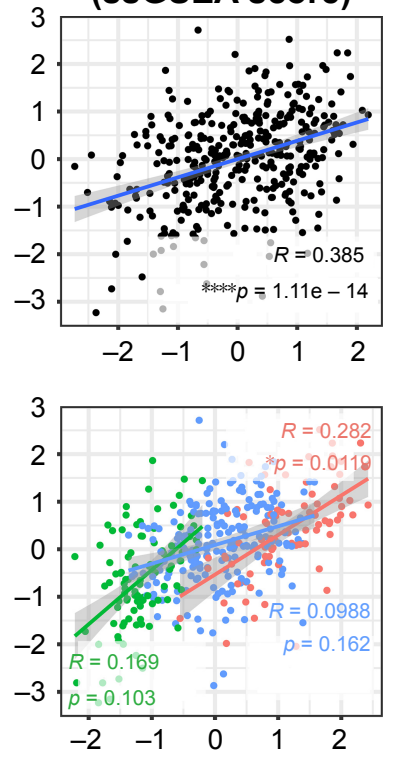

Treg (ssGSEA score)
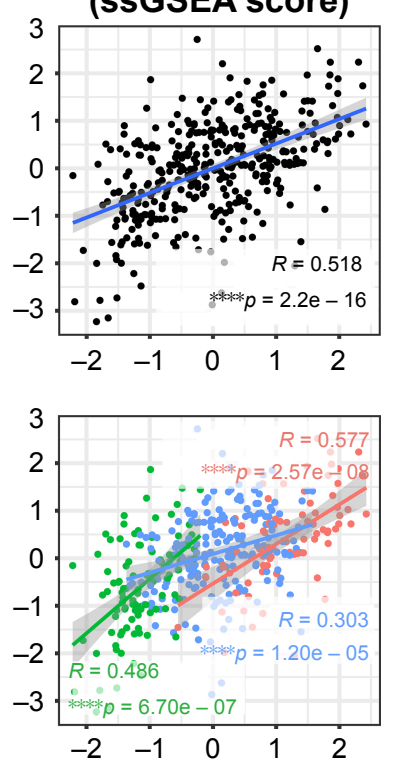

$\longrightarrow$ High Median $\longrightarrow$ Low

B

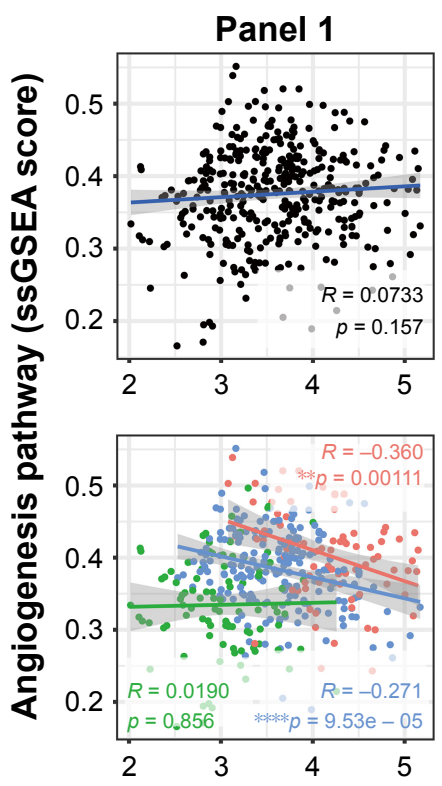

IFN- $\gamma$ signature
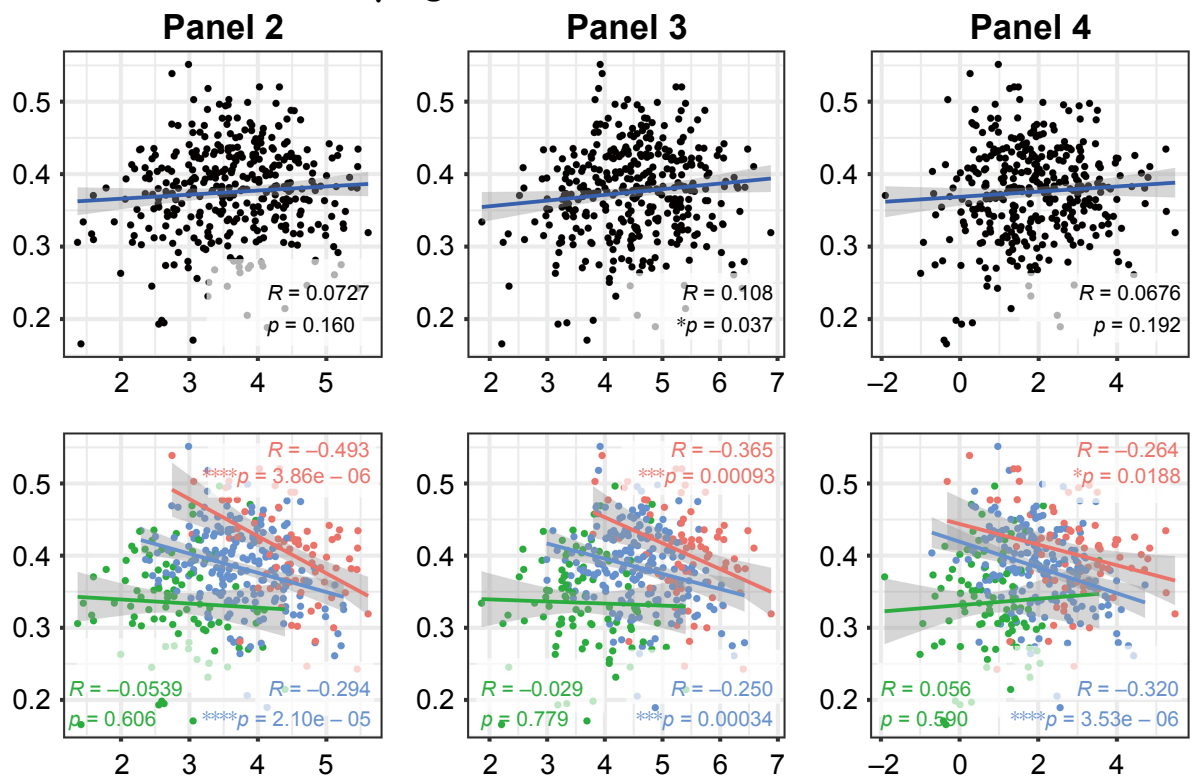

\section{$\longrightarrow$ High $\longrightarrow$ Median $\longrightarrow$ Low}

Figure 3 Correlations between immune signatures and the angiogenesis pathway.

Notes: (A) Correlations between immune-activating and immune-inhibitory signatures and the angiogenesis pathway. (B) Correlations between angiogenesis and different panels of IFN- $\gamma$ signatures from KEYNOTE-059 (panel I), CheckMate-275 (panel 2), KEYNOTE-0I2 (panel 3), and POPLAR (panel 4). All $r$ values are Pearson's correlation coefficients. Two-tailed $p$-values are presented to indicate significance $(<0.05 ; \mathrm{N}=375)$. ${ }^{*} p<0.05, * * p<0.005$, $* * * p<0.001$, $* * * * p<0.000 \mathrm{I}$.

Abbreviations: ssGSEA, single-sample gene set enrichment analysis; CYT, cytotoxic; MDSC, myeloid-derived suppressor cell.

of pembrolizumab in gastric cancers were reported in KEYNOTE-012, ${ }^{3}$ KEYNOTE-028, ${ }^{30}$ and KEYNOTE-059 studies, ${ }^{13}$ with an ORR of $22 \%, 30.4 \%$, and $13.3 \%$, respectively. These exciting results made pembrolizumab the first third-line therapy for advanced gastric cancer approved by the US Food and Drug Administration (FDA). Another PD-1 antibody nivolumab also exhibited encouraging clinical activities. ${ }^{31}$ Nevertheless, the results of monotherapy with ICIs in gastric cancer may not yet be as impressive as those seen in melanoma, ${ }^{32}$ lung, ${ }^{15}$ and kidney cancers. ${ }^{14}$ The 
A
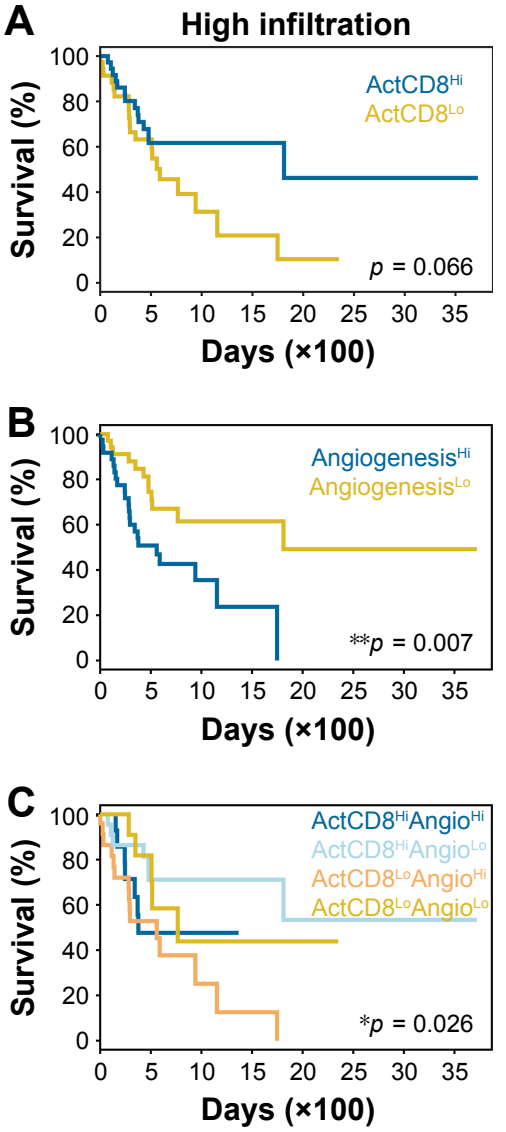
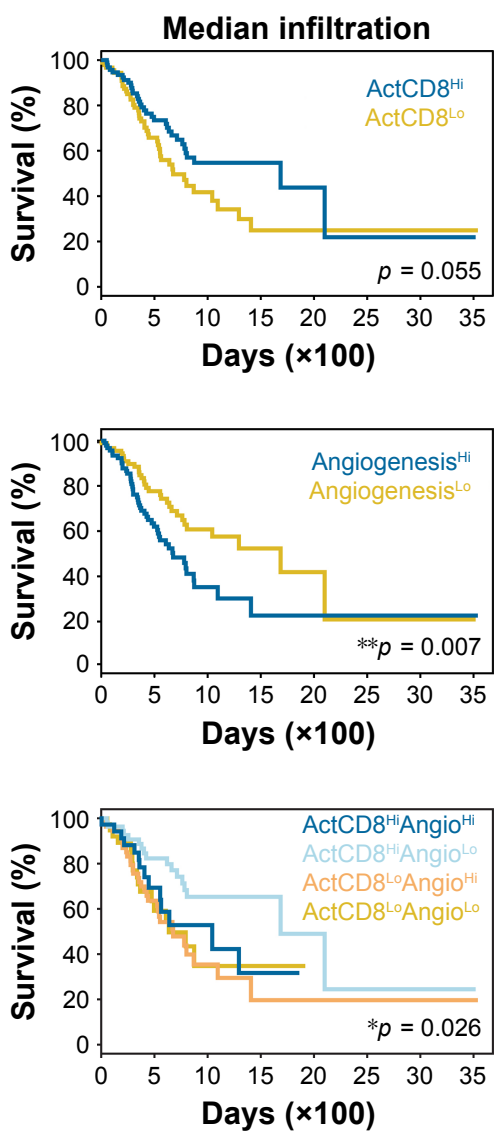

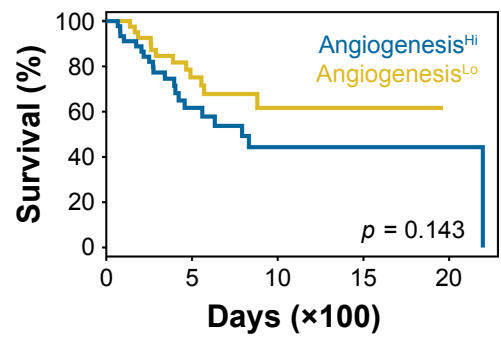

Low infiltration
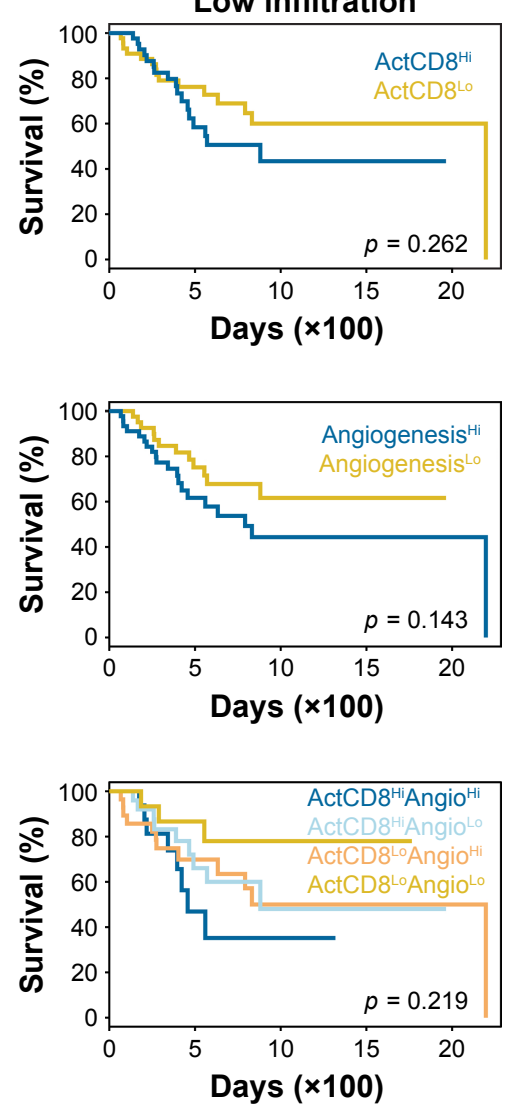

Figure 4 Prognosis predictions by angiogenesis and infiltration of activated $\mathrm{CD} 8^{+} \mathrm{T}$ cells.

Notes: (A) Survival curves for different infiltration phenotypes between groups categorized by infiltration of activated CD8 $8^{+}$cells. (B) Survival curves for different infiltration phenotypes between groups categorized by the angiogenesis pathway from Hallmark gene sets. (C) Dual-factor predictions of survival in different infiltration groups, utilizing combinations of infiltration of activated CD8 ${ }^{+} \mathrm{T}$ cells and angiogenesis. $p$-values for significance $(<0.05)$ were determined using multivariable Cox regression analysis $(\mathrm{N}=375)$. ${ }_{p}<0.05,{ }^{* *} p<0.005$.

effective response to ICIs in patients with gastric cancer has been associated with high PD-L1 expression, microsatellite instability, and high IFN- $\gamma$ signature score; ${ }^{3,13}$ these characteristics can only be found in a minor proportion of patients, which is a major limitation to providing clinical benefit to the entire population. Therefore, enhancing the efficacy of ICIs in gastric cancers has become an imperative issue.

The aforementioned characteristics are usually related to immune characterization in the TME, suggesting that ameliorating the TME may improve the ORR of ICIs. The constitution of TME is attributed to a multitude of tumor-intrinsic and tumor-extrinsic mechanisms, wherein angiogenesis plays an important role. Pathologic angiogenesis may influence antitumor immunity in the following ways: 1) impeding lymphocytic migration to tumor loci via hypoxia-induced downregulation of adhesion molecules; ${ }^{33,34}$ 2) inducing immune tolerance and anergy by overexpression of immune-inhibitory molecules via endothelial cells in the tumor vasculature; 35,363 ) directly regulating immune cells through VEGFA, HIF-1 $\alpha$, and other factors. ${ }^{4,37}$ In our study, the potent impact of angiogenesis on the TME of gastric cancers was validated by machine learning, using RNA sequencing data in a large cohort of gastric cancers from the TCGA database. The direct association between angiogenesis and immune infiltration was further demonstrated by correlation analysis. Our data highlighted the essential role of angiogenesis in determining TME immune characterization. Meanwhile, we also demonstrated the unessential role of $\mathrm{Wnt} / \beta$-catenin signaling, different from discoveries in melanoma, ${ }^{23}$ suggesting a tumor-type-dependent mechanism of tuning immune cell infiltration.

Clinical trials on antiangiogenic drugs, including ramucirumab and apatinib, have achieved encouraging outcomes. ${ }^{6-8}$ Considering the determinant role of angiogenesis in the immune niche, it is reasonable to believe that combination of immunotherapy and antiangiogenesis will result in a favorable prognosis. Such a treatment strategy is under investigation in other solid tumors and has verified the reinvigoration of immune infiltration, as well as impressively improved response. ${ }^{38-41}$ Our data also indicated the 
potential benefit of combination therapy for patients with gastric cancers, particularly for those with inflamed immune characterization.

Nevertheless, all conclusions herein were drawn at the sequencing level and thus are still theoretical; our observations require future validation by clinical investigations. In addition, prolonged survival of patients with low angiogenesis signaling and high CTL infiltration was not observed in the low infiltration group, implying that there may be other targets for patients with extremely impaired antitumor immunity. If potential mechanisms defining the prognoses of immune-inhibitory population are resolved clinically, there will be additional clinical benefits available for a greater number of patients.

\section{Conclusion}

Our study identified the distinct infiltration landscapes of gastric cancers, which were significantly associated with angiogenesis, the most targetable signaling pathway. The relationship between angiogenesis and the tumor immune microenvironment suggested that targeting angiogenesis may ameliorate the immune niche and provide a rationale for combination therapy and subsequent clinical investigations in gastric cancer.

\section{Acknowledgment}

This study was funded by the National Natural Science Foundation of China (grant number: 81222031).

\section{Author contributions}

$\mathrm{QJ}, \mathrm{YF}$, and BZ conceived and designed the experiments. YD, J-NC, and CS collected public data and directed the cohort studies. ZG, XZ, LZ, and YF wrote scripts for data analysis. YF and QJ analyzed and interpreted data. YF, QJ, and BZ wrote the manuscript. All authors contributed toward data analysis, drafting and revising the paper and agree to be accountable for all aspects of the work. All authors read and approved the final manuscript.

\section{Disclosure}

The authors report no conflicts of interest in this work.

\section{References}

1. Ferlay J, Soerjomataram I, Dikshit R, et al. Cancer incidence and mortality worldwide: sources, methods and major patterns in GLOBOCAN 2012. Int J Cancer. 2015;136(5):E359-E386.

2. Gentzler R, Hall R, Kunk PR, et al. Beyond melanoma: inhibiting the PD-1/PD-L1 pathway in solid tumors. Immunotherapy. 2016;8(5): 583-600.
3. Muro K, Chung HC, Shankaran V, et al. Pembrolizumab for patients with PD-L1-positive advanced gastric cancer (KEYNOTE-012): a multicentre, open-label, phase 1b trial. Lancet Oncol. 2016;17(6): 717-726.

4. Voron T, Colussi O, Marcheteau E, et al. VEGF-A modulates expression of inhibitory checkpoints on CD8+ T cells in tumors. J Exp Med. 2015;212(2):139-148.

5. Ohtsu A, Shah MA, Van Cutsem E, et al. Bevacizumab in combination with chemotherapy as first-line therapy in advanced gastric cancer: a randomized, double-blind, placebo-controlled phase III study. J Clin Oncol. 2011;29(30):3968-3976.

6. Fuchs CS, Tomasek J, Yong CJ, et al. Ramucirumab monotherapy for previously treated advanced gastric or gastro-oesophageal junction adenocarcinoma (REGARD): an international, randomised, multicentre, placebo-controlled, phase 3 trial. Lancet. 2014;383(9911):31-39.

7. Wilke H, Muro K, Van Cutsem E, et al. Ramucirumab plus paclitaxel versus placebo plus paclitaxel in patients with previously treated advanced gastric or gastro-oesophageal junction adenocarcinoma (RAINBOW): a double-blind, randomised phase 3 trial. Lancet Oncol. 2014;15(11):1224-1235.

8. Li J, Qin S, Xu J, et al. Apatinib for chemotherapy-refractory advanced metastatic gastric cancer: results from a randomized, placebo-controlled, parallel-arm, phase II trial. J Clin Oncol. 2013;31(26):3219-3225.

9. Hanzelmann S, Castelo R, Guinney J. GSVA: gene set variation analysis for microarray and RNA-seq data. BMC Bioinformatics. 2013;14:7.

10. Barbie DA, Tamayo P, Boehm JS, et al. Systematic RNA interference reveals that oncogenic KRAS-driven cancers require TBK1. Nature. 2009;462(7269): 108-112.

11. Rooney MS, Shukla SA, Wu CJ, Getz G, Hacohen N. Molecular and genetic properties of tumors associated with local immune cytolytic activity. Cell. 2015;160(1-2):48-61.

12. Mandal R, Senbabaoglu Y, Desrichard A, et al. The head and neck cancer immune landscape and its immunotherapeutic implications. JCI Insight. 2016;1(17):e89829.

13. Fuchs CS, Doi T, Jang RW-J, et al. KEYNOTE-059 cohort 1: efficacy and safety of pembrolizumab (pembro) monotherapy in patients with previously treated advanced gastric cancer. J Clin Oncol. 2017; 35(15_suppl):4003.

14. Sharma P, Retz M, Siefker-Radtke A, et al. Nivolumab in metastatic urothelial carcinoma after platinum therapy (CheckMate 275): a multicentre, single-arm, phase 2 trial. Lancet Oncol. 2017;18(3):312-322.

15. Fehrenbacher L, Spira A, Ballinger M, et al. Atezolizumab versus docetaxel for patients with previously treated non-small-cell lung cancer (POPLAR): a multicentre, open-label, phase 2 randomised controlled trial. Lancet. 2016;387(10030):1837-1846.

16. Charoentong P, Finotello F, Angelova M, et al. Pan-cancer immunogenomic analyses reveal genotype-immunophenotype relationships and predictors of response to checkpoint blockade. Cell Rep. 2017; 18(1):248-262.

17. Angelova M, Charoentong P, Hackl H, et al. Characterization of the immunophenotypes and antigenomes of colorectal cancers reveals distinct tumor escape mechanisms and novel targets for immunotherapy. Genome Biol. 2015;16:64.

18. Breiman L. Random forests. Mach Learn. 2001;45(1):5-32.

19. Ji H, Houghton AM, Mariani TJ, et al. K-ras activation generates an inflammatory response in lung tumors. Oncogene. 2006;25(14): $2105-2112$.

20. Okumura T, Ericksen RE, Takaishi S, et al. K-ras mutation targeted to gastric tissue progenitor cells results in chronic inflammation, an altered microenvironment, and progression to intraepithelial neoplasia. Cancer Res. 2010;70(21):8435-8445.

21. Gaiha GD, McKim KJ, Woods M, et al. Dysfunctional HIV-specific $\mathrm{CD} 8+\mathrm{T}$ cell proliferation is associated with increased caspase- 8 activity and mediated by necroptosis. Immunity. 2014;41(6):1001-1012.

22. Pereira-Manfro WF, Ribeiro-Gomes FL, Filardy AA, et al. Inhibition of caspase-8 activity promotes protective Th1- and Th2-mediated immunity to Leishmania major infection. J Leukoc Biol. 2014;95(2):347-355. 
23. Spranger S, Bao R, Gajewski TF. Melanoma-intrinsic beta-catenin signalling prevents anti-tumour immunity. Nature. 2015;523(7559): 231-235.

24. Allen E, Jabouille A, Rivera LB, et al. Combined antiangiogenic and anti-PD-L1 therapy stimulates tumor immunity through HEV formation. Sci Transl Med. 2017;9(385):eaak9679.

25. Hendry SA, Farnsworth RH, Solomon B, Achen MG, Stacker SA, Fox SB. The role of the tumor vasculature in the host immune response: implications for therapeutic strategies targeting the tumor microenvironment. Front Immunol. 2016;7:621.

26. Horikawa N, Abiko K, Matsumura N, et al. Expression of vascular endothelial growth factor in ovarian cancer inhibits tumor immunity through the accumulation of myeloid-derived suppressor cells. Clin Cancer Res. 2017;23(2):587-599.

27. Staal FJ, Luis TC, Tiemessen MM. WNT signalling in the immune system: WNT is spreading its wings. Nat Rev Immunol. 2008;8(8): 581-593.

28. Hugo W, Zaretsky JM, Sun L, et al. Genomic and transcriptomic features of response to anti-PD-1 therapy in metastatic melanoma. Cell. 2016; 165(1):35-44.

29. Ralph C, Elkord E, Burt DJ, et al. Modulation of lymphocyte regulation for cancer therapy: a phase II trial of tremelimumab in advanced gastric and esophageal adenocarcinoma. Clin Cancer Res. 2010;16(5): 1662-1672.

30. Doi T, Piha-Paul SA, Jalal SI, et al. Updated results for the advanced esophageal carcinoma cohort of the phase 1b KEYNOTE-028 study of pembrolizumab. J Clin Oncol. 2016;34(15_suppl):4046.

31. Janjigian YY, Bendell JC, Calvo E, et al. CheckMate-032: phase I/II, open-label study of safety and activity of nivolumab (nivo) alone or with ipilimumab (ipi) in advanced and metastatic (A/M) gastric cancer (GC). J Clin Oncol. 2016;34(15_suppl):4010.
32. Ribas A, Hamid O, Daud A, et al. Association of pembrolizumab with tumor response and survival among patients with advanced melanoma. JAMA. 2016;315(15):1600-1609.

33. Tromp SC, oude Egbrink MG, Dings RP, et al. Tumor angiogenesis factors reduce leukocyte adhesion in vivo. Int Immunol. 2000;12(5): 671-676.

34. Horsman MR, Vaupel P. Pathophysiological basis for the formation of the tumor microenvironment. Front Oncol. 2016;6:66.

35. Choi J, Enis DR, Koh KP, Shiao SL, Pober JS. T lymphocyte-endothelial cell interactions. Annu Rev Immunol. 2004;22:683-709.

36. Carman CV, Martinelli R. T lymphocyte-endothelial interactions: emerging understanding of trafficking and antigen-specific immunity. Front Immunol. 2015;6:603.

37. Facciabene A, Peng X, Hagemann IS, et al. Tumour hypoxia promotes tolerance and angiogenesis via CCL28 and T(reg) cells. Nature. 2011;475(7355):226-230.

38. Hodi FS, Lawrence D, Lezcano C, et al. Bevacizumab plus ipilimumab in patients with metastatic melanoma. Cancer Immunol Res. 2014; 2(7):632-642.

39. Wu X, Giobbie-Hurder A, Liao X, et al. VEGF neutralization plus CTLA-4 blockade alters soluble and cellular factors associated with enhancing lymphocyte infiltration and humoral recognition in melanoma. Cancer Immunol Res. 2016;4(10):858-868.

40. Wallin JJ, Bendell JC, Funke R, et al. Atezolizumab in combination with bevacizumab enhances antigen-specific T-cell migration in metastatic renal cell carcinoma. Nat Commun. 2016;7:12624.

41. Nakahama K, Isa SI, Tamiya A, et al. The association between chemotherapy immediately before nivolumab and outcomes thereafter. Anticancer Res. 2017;37(10):5885-5891.
OncoTargets and Therapy

\section{Publish your work in this journal}

OncoTargets and Therapy is an international, peer-reviewed, open access journal focusing on the pathological basis of all cancers, potential targets for therapy and treatment protocols employed to improve the management of cancer patients. The journal also focuses on the impact of management programs and new therapeutic agents and protocols on

\section{Dovepress}

patient perspectives such as quality of life, adherence and satisfaction. The manuscript management system is completely online and includes a very quick and fair peer-review system, which is all easy to use. Visit http://www.dovepress.com/testimonials.php to read real quotes from published authors. 